\title{
SMALL REYNOLDS NUMBER FLOW BETWEEN ECCENTRIC ROTATING CYLINDERS WITH A PERMEABLE SLEEVE
}

\author{
L. D. HIRD and P. F. SIEW ${ }^{1}$
}

(Received 22 August 1994; revised 16 February 1995)

\begin{abstract}
Two eccentric rotating cylinders together with a permeable membrane surrounding the inner cylinder are used to model the flow around a modified viscometer. A perturbation method is used to solve for the flow between the membrane and the outer cylinder; the flow between the inner rotor and the membrane is assumed to be governed by Stoke's equation, and the two flow regimes are coupled by the through-flow across the membrane. For moderate values of Reynolds number and eccentricity, the permeability of the membrane plays a negligible role, and the flow through the membrane is found to be eccentricity dependent. High eccentricities result in the formation of eddies which, upon increasing the Reynolds number, move in a direction opposite to that of the rotation of the outer bowl.
\end{abstract}

\section{Introduction}

The problem outlined in this paper arises from an attempt to model the fluid flow around a modified viscometer used for slurries that have a tendency to settle. Briefly, the viscometer consists of a rotor surrounded by a fixed cylinder or sleeve. The sleeve is either slotted or perforated allowing fluid to flow through from the outside. The assembly is lowered into an outer bowl, whose rotation ensures the slurry is mixed continuously, and the slotted or perforated sleeve facilitates the entrainment of the well mixed slurry onto the surface of the rotor. The rotational speed of the rotor can be set independently so that the fluids shear rate can be varied continuously from zero to some chosen maximum whilst the shear stress on the rotor is measured. The plot of the shear stress against the shear rate produces a rheogram from which the constitutive relation of the fluid may be deduced. Although this technique appears to offer an effective means of estimating the rheological properties of settling slurries, there are still questions regarding the accuracy of the results when parameters (such as rates of rotation, distribution of perforations on the sleeve, etc) are changed or when vorticies

${ }^{1}$ Curtin University of Technology, GPO Box U1987, Perth Western Australia 6001, Australia

(C) Australian Mathematical Society, 1996, Serial-fee code 0334-2700/96 
are present within the bowl (Overend et al. [4]). The properties of some slurries (for example, clay slurries) may be approximated by a Bingham fluid, but numerical techniques appear to be the only effective way to study this problem. The present paper does not attempt to give any quantitative answers to the problem; however, as a first step, we propose to consider the simpler case of a Newtonian fluid, since, apart from experimental observations, little is known about the flow pattern around the viscometer. The actual problem is three dimensional; nevertheless, if we assume that the bowl is large enough and the viscometer is constructed so that end effects are minimized then a two dimensional model can be considered and a perturbation scheme used.



FIGURE 1. Geometry and coordinate systems.

We concentrate, therefore, on the steady flow of a viscous homogeneous fluid in a cylinder of radius $b$, rotating with angular velocity $\Omega$ [see Figure 1]. A viscometer consisting of a rotor of radius $c$ rotating with angular velocity $\omega$ and surrounded by a sleeve (a stationary concentric cylinder of radius $a$ ) is situated with centre a small distance from the centre of the rotating outer cylinder. The actual sleeve has a small, but finite thickness, and its small perforations will restrict any tangential component 
of velocity in the fluid flowing through it. In order to make the problem amenable to analytical techniques we make two assumptions: firstly, the distance between the two centres $a \epsilon$ say, is assumed to be small; however, this assumption will be relaxed later, as explained in Section 4; secondly, the perforated or slotted sleeve is replaced by a non-deformable permeable membrane (of zero thickness) over which a no-slip condition is applied. The problem can, therefore, be divided into an outer problem involving the flow between the membrane and the outer cylinder and an inner problem between the rotor and the membrane. Furthermore, if we assume that the flux across the membrane is proportional to the pressure difference across it, which has been shown by Woods [8] to be an adequate model for the flow across a perforated surface whose perforations are small and uniformly distributed, then the two regimes could be coupled by this condition.

\section{The outer problem}

Here, we are concerned with the flow of a viscous fluid between eccentric cylinders where the outer cylinder is rotating. This problem has been studied by various authors in different context. Wood [7] used an asymptotic technique to study the flow in the limit of large Reynolds numbers, and DiPrima and Stuart [3] considered the low Reynolds number limit for the flow in journal bearings. While many other works deal with the case of two eccentric cylinders, the addition of a permeable surface makes the problem unique.

We use the conformal transformation outlined by Wood [7] to map the two cylinders for the outer problem onto the concentric cylinders with radii given by $\rho=1$ and $\rho=\beta$ where $\beta>1$. The defining equations are

$$
z=\frac{a(w+\gamma)}{1+\gamma w}, \quad z=r e^{i \theta}, \quad w=\rho e^{i \phi},
$$

where

$$
\gamma=-2 \epsilon\left[(b / a)^{2}-1-\epsilon^{2}+\sqrt{\left(b^{2} / a^{2}-1-\epsilon^{2}\right)^{2}-4 \epsilon^{2}}\right]^{-1},
$$

and

$$
\beta=\frac{(b / a)}{1-\epsilon \gamma} .
$$

(This simple relation for $\beta$ is equivalent to that given by Wood.)

A stream function, $\psi$, may be defined in terms of the non-dimensional velocity components given by

$$
u_{\rho}=\frac{\sqrt{J}}{\rho} \frac{\partial \psi}{\partial \phi}, \quad u_{\phi}=-\sqrt{J} \frac{\partial \psi}{\partial \rho},
$$


where the Jacobian of the transformation is

$$
J=\frac{\left(1+2 \gamma \rho \cos \phi+\gamma^{2} \rho^{2}\right)^{2}}{\left(1-\gamma^{2}\right)^{2}} .
$$

Taking $a$ as the length scale (which is implied by (2.1)), $b \Omega$ as a unit of velocity and eliminating the pressure from the equations of motion, we obtain

$$
-\frac{1}{\rho} \frac{\partial(\psi, \zeta)}{\partial(\rho, \phi)}=\frac{1}{R} \nabla^{2} \zeta, \quad \zeta=-J \nabla^{2} \psi
$$

where

$$
\nabla^{2}=\frac{\partial^{2}}{\partial \rho^{2}}+\frac{1}{\rho} \frac{\partial}{\partial \rho}+\frac{1}{\rho^{2}} \frac{\partial^{2}}{\partial \phi^{2}}
$$

Here, $\zeta$ is the axial component of vorticity and $R=a b \Omega / \nu$ is the Reynolds number with kinematic viscosity $\nu$. Since $\epsilon$ is small, $\gamma$ as given by (2.2) is also a small parameter. In the limit as $\epsilon \rightarrow 0, \gamma \rightarrow 0, J \rightarrow 1$ and the outer problem becomes the solution, which is known to be independent of the Reynolds number, given by that of axi-symmetric flow between concentric cylinders. In this limit, we expect no interaction between the two flow regimes. At the permeable membrane, the normal flux is assumed to be driven by the variation of the pressure difference, and the conservation of mass requires that the total flow across it is zero. Thus, if $f(\phi ; \gamma)$ denotes the normal velocity distribution at $\rho=1$ then it has a period of $2 \pi$ in $\phi$ and must satisfy

$$
u_{\rho}(1, \phi)=f(\phi ; \gamma), \quad \int_{-\pi}^{\pi} f(\phi ; \gamma) / \sqrt{J(1, \phi)} d \phi=0
$$

The boundary conditions for the outer problem become

$$
\left.\begin{array}{llrl}
\frac{\partial \psi}{\partial \rho}(\beta, \phi) & =-\frac{1}{\sqrt{J(\beta, \phi)}}, & \psi(\beta, \phi) & =\text { constant } \\
\frac{\partial \psi}{\partial \rho}(1, \phi) & =0, & \frac{\partial \psi}{\partial \phi}(1, \phi) & =f(\phi ; \gamma) / \sqrt{J(1, \phi)} .
\end{array}\right\} .
$$

2.1. A perturbation scheme Following Wood [7] we look at a perturbation scheme for $\psi$ and $\zeta$ given by

$$
\left.\begin{array}{rl}
\psi & =\psi_{0}(\rho)+\sum_{n=1}^{\infty} \gamma^{n} \psi_{n}(\rho, \phi ; R) \\
\left.\gamma^{2}\right)^{2} \zeta & =\zeta_{0}+\sum_{n=1}^{\infty} \gamma^{n} \zeta_{n}(\rho, \phi ; R) .
\end{array}\right\}
$$


The axi-symmetric zeroth order solution can be written down at once, being

$$
\psi_{0}(\rho)=-\frac{1}{2} A \rho^{2}-B \log \rho+K,
$$

where $A, B$ and $K$ are constants. For flows between concentric cylinders, the constant $K$ is immaterial and is normally equated to zero; however, for the present problem it can be determined only from a knowledge of the solution to the inner problem since its value depends on the relative flow between the boundaries of the two regimes. As expected, this solution does not contribute to the flux across the permeable membrane. In view of this, the velocity distribution, given by (2.7), may be written as

$$
f(\phi ; \gamma)=\gamma f_{1}(\phi)+\gamma^{2} f_{2}(\phi)+\cdots .
$$

Expanding the boundary conditions given in (2.8) in powers of $\gamma$, we obtain

$$
\frac{\partial \psi_{0}}{\partial \rho}(1, \phi)=0, \quad \frac{\partial \psi_{0}}{\partial \rho}(\beta, \phi)=-1, \quad \psi_{0}(\beta, \phi)=\text { constant }
$$

for the zeroth order term. Equations (2.10) and (2.12) now yield

$$
\psi_{0}=-A\left(\frac{1}{2} \rho^{2}-\log \rho\right)+K, \quad \zeta_{0}=2 A, \quad A=\beta /\left(\beta^{2}-1\right) .
$$

The corresponding boundary conditions for the first order terms are

$$
\left.\begin{array}{rlrl}
\frac{\partial \psi_{1}}{\partial \phi}(1, \phi) & =f_{1}(\phi), & \frac{\partial \psi_{1}}{\partial \rho}(1, \phi) & =0 \\
\frac{\partial \psi_{1}}{\partial \rho}(\beta, \phi) & =2 \beta \cos \phi, & \psi_{1}(\beta, \phi) & =0
\end{array}\right\}
$$

and from (2.6) we have

$$
-\frac{1}{\rho} \frac{\partial \psi_{0}}{\partial \rho} \frac{\partial \zeta_{1}}{\partial \phi}=\frac{1}{R} \nabla^{2} \zeta_{1}, \quad \zeta_{1}=-\nabla^{2}\left(\psi_{1}-A \rho^{3} \cos \phi\right) .
$$

The governing equations and the boundary conditions for the second order terms are

$$
\begin{gathered}
-\frac{1}{\rho}\left[\frac{\partial \psi_{0}}{\partial \rho} \frac{\partial \zeta_{2}}{\partial \phi}+\frac{\partial\left(\psi_{1}, \zeta_{1}\right)}{\partial(\rho, \phi)}\right]=\frac{1}{R} \nabla^{2} \zeta_{2}, \\
\zeta_{2}=4 \rho \cos \phi \zeta_{1}+4 \rho^{2} \zeta_{0}-\nabla^{2}\left(\psi_{2}+2 A \rho^{4} \cos ^{2} \phi\right),
\end{gathered}
$$

and

$$
\left.\begin{array}{ll}
\frac{\partial \psi_{2}}{\partial \phi}(1, \phi)=f_{2}(\phi)-2 f_{1}(\phi) \cos \phi, & \frac{\partial \psi_{2}}{\partial \rho}(1, \phi)=0, \\
\frac{\partial \psi_{2}}{\partial \rho}(\beta, \phi)=1+\beta^{2}-4 \beta^{2} \cos ^{2} \phi, & \psi_{2}(\beta, \phi)=0 .
\end{array}\right\}
$$


The equations for the higher order terms are easily generated if required. Equation (2.16) does not contain any coefficients of large magnitude, as can be verified once the form of $\psi_{1}(\rho, \phi)$ is known; hence, for small values of $|\gamma|$ we expect the zero and first order solutions to give a good description of the flow pattern.

2.1.1. The first order solution We now assume that $f_{1}(\phi)$ may be expressed as a Fourier series of the form

$$
f_{1}(\phi)=\sum_{n=1}^{\infty} a_{n} \cos n \phi+b_{n} \sin n \phi .
$$

In practice, $f_{1}(\phi)$ is approximated to a high degree of accuracy by a finite number of terms. This, and (2.15), suggests that we can let

$$
\psi_{1}(\rho, \phi ; R)=A \rho^{3} \cos \phi+\Re\left(\sum_{n=1}^{\infty} \chi_{n}(\rho) e^{i n \phi}\right),
$$

and

$$
\zeta_{1}(\rho, \phi ; R)=-\Re\left(\nabla^{2}\left[\sum_{n=1}^{\infty} \chi_{n}(\rho) e^{i n \phi}\right]\right),
$$

where $\Re$ denotes the real part of the expression. If

$$
\zeta_{1}(\rho, \phi ; R)=\Re\left\{\sum_{n=1}^{\infty} \xi_{n}(\rho) e^{i n \phi}\right\}
$$

then $(2.15)$ yields

$$
\xi_{n}^{\prime \prime}+\frac{1}{\rho} \xi_{n}^{\prime}-\frac{n^{2}}{\rho^{2}} \xi_{n}=\operatorname{inAR}\left(1-\frac{1}{\rho^{2}}\right) \xi_{n},
$$

where the prime denotes differentiation with respect to $\rho$, and

$$
\chi_{n}^{\prime \prime}+\frac{1}{\rho} \chi_{n}^{\prime}-\frac{n^{2}}{\rho^{2}} \chi_{n}=-\xi_{n} .
$$

(When $n=1$, we have the set of equations used by Wood.) The boundary conditions in (2.14) are now replaced by

$$
\left.\begin{array}{ll}
\chi_{n}(1)=-\epsilon_{n} A-\frac{i}{n \pi} \int_{-\pi}^{\pi} f_{1}(\phi) e^{-i n \phi} d \phi, & \chi_{n}^{\prime}(1)=-3 A \epsilon_{n}, \\
\chi_{n}^{\prime}(\beta)=\left(2 \beta-3 A \beta^{2}\right) \epsilon_{n}, & \chi_{n}(\beta)=-A \beta^{3} \epsilon_{n},
\end{array}\right\}
$$


where $\epsilon_{1}=1$, and $\epsilon_{n}=0$ for $n \neq 1$. Writing $\mu_{n}=\sqrt{n^{2}-i n A R}$ and $\lambda_{n}=\sqrt{i n A R}$, the solution to $(2.22)$ is

$$
\xi_{n}(\rho)=C_{n} I_{\mu_{n}}\left(\lambda_{n} \rho\right)+D_{n} I_{-\mu_{n}}\left(\lambda_{n} \rho\right),
$$

where $C_{n}$ and $D_{n}$ are arbitrary constants to be determined and $I_{ \pm \mu_{n}}$ are the modified Bessel functions. We note that

$$
\begin{aligned}
I_{ \pm \mu_{n}}\left(\lambda_{n} \rho\right) & =I_{ \pm \mu_{n}}\left(e^{i \pi / 4} \sqrt{n A R}\right) \\
& =\exp \left(\mp i \pi \mu_{n} / 2\right) J_{ \pm \mu_{n}}\left(e^{i 3 \pi / 4} \sqrt{n A R}\right)
\end{aligned}
$$

which may in turn be written in terms of the Thomson functions $\left(\right.$ ber $_{\mu_{n}}$ and $\left.b e i_{\mu_{n}}\right)$. When $n$ is large the Bessel function $J_{\mu_{n}}\left(e^{i 3 \pi / 4} \sqrt{n A R}\right)$ may be approximated by use of Meissel's formula (see [6, p. 227]). Substituting (2.25) into (2.23) and solving leads to

$$
\chi_{n}(\rho)=A_{n} \rho^{n}+\frac{B_{n}}{\rho^{n}}-C_{n} \mathscr{I}_{n}^{(1)}(\rho)-D_{n} \mathscr{I}_{n}^{(2)}(\rho),
$$

where $A_{n}$ and $B_{n}$ are arbitrary constants, and

$$
\mathscr{I}_{n}^{(1)}(\rho)=\frac{1}{2 n} \int_{1}^{\rho}\left(\frac{\rho^{n}}{s^{n-1}}-\frac{s^{n+1}}{\rho^{n}}\right) I_{\mu_{n}}\left(\lambda_{n} s\right) d s,
$$

and

$$
\mathscr{I}_{n}^{(2)}(\rho)=\frac{1}{2 n} \int_{\rho}^{\beta}\left(\frac{s^{n+1}}{\rho^{n}}-\frac{\rho^{n}}{s^{n-1}}\right) I_{-\mu_{n}}\left(\lambda_{n} s\right) d s .
$$

By construction, $\mathscr{I}_{n}^{(1)}(1)=0=\mathscr{I}_{n}^{(1)^{\prime}}(1)$ and $\mathscr{I}_{n}^{(2)}(\beta)=0=\mathscr{I}_{n}^{(2)^{\prime}}(\beta) . A_{n}, B_{n}, C_{n}$ and $D_{n}$ can now be determined using (2.24). Writing

$$
\begin{aligned}
\Delta_{n}= & \left(\beta^{2 n}-1\right)\left\{\beta \mathscr{I}_{n}^{(1)^{\prime}}(\beta) \mathscr{I}_{n}^{(2)^{\prime}}(1)-n^{2} \mathscr{I}_{n}^{(1)}(\beta) \mathscr{I}_{n}^{(2)}(1)\right\} \\
& +n\left(\beta^{2 n}+1\right)\left\{\beta \mathscr{I}_{n}^{(1)^{\prime}}(\beta) \mathscr{I}_{n}^{(2)}(1)-\mathscr{I}_{n}^{(1)}(\beta) \mathscr{I}_{n}^{(2)^{\prime}}(1)\right\},
\end{aligned}
$$

we have

$$
\begin{aligned}
\Delta_{n} C_{n}= & 4 \beta^{2} \mathscr{I}_{1}^{(2)}(1) \epsilon_{n}-2 n \beta^{n} E_{n} \mathscr{I}_{n}^{(2)^{\prime}}(1), \\
\Delta_{n} D_{n}= & {\left[2 \beta\left(\beta^{2}+1\right) \mathscr{I}_{1}^{(1)}(\beta)-2 \beta^{2}\left(\beta^{2}-1\right) \mathscr{I}_{1}^{(1)^{\prime}}(\beta)\right] \epsilon_{n} } \\
& +n E_{n}\left[\left(\beta^{2 n}-1\right) n \mathscr{I}_{n}^{(1)}(\beta)-\beta\left(\beta^{2 n}+1\right) \mathscr{I}_{n}^{(1)^{\prime}}(\beta)\right], \\
A_{n}= & -2 \beta /\left(\beta^{2}-1\right) \epsilon_{n}+E_{n} / 2+\left(D_{n} / 2\right)\left\{\mathscr{I}_{n}^{(2)}(1)+\frac{1}{n} \mathscr{I}_{n}^{(2)^{\prime}}(1)\right\}, \\
B_{n}= & \beta /\left(\beta^{2}-1\right) \epsilon_{n}+E_{n} / 2+\left(D_{n} / 2\right)\left\{\mathscr{I}_{n}^{(2)}(1)-\frac{1}{n} \mathscr{I}_{n}^{(2)^{\prime}}(1)\right\},
\end{aligned}
$$


and

$$
E_{n}=-\frac{i}{n \pi} \int_{-\pi}^{\pi} f_{1}(\phi) e^{-i n \phi} d \phi=-\left(i a_{n}+b_{n}\right) / n
$$

From the Navier Stokes equation, and assuming the expansion schemes

$$
\left.\begin{array}{l}
\Pi(\rho, \phi)=\sum_{n=0}^{\infty} \gamma^{n} \Pi_{n}(\rho, \phi), \\
u_{\rho}(\rho, \phi)=\sum_{n=1}^{\infty} \gamma^{n} u_{n}(\rho, \phi), \\
u_{\phi}(\rho, \phi)=\sum_{n=0}^{\infty} \gamma^{n} v_{n}(\rho, \phi),
\end{array}\right\}
$$

for the pressure $\Pi(\rho, \phi)$ and the velocity components $u_{\rho}(\rho, \phi)$ and $u_{\phi}(\rho, \phi)$, the first two terms for the pressure distribution are given by

$$
\frac{\partial \Pi_{0}}{\partial \phi}=0
$$

and

$$
\frac{\partial \Pi_{1}}{\partial \phi}=-\rho u_{1} \frac{\partial v_{0}}{\partial \rho}-v_{0} \frac{\partial v_{1}}{\partial \phi}-v_{0} u_{1}+\frac{\rho}{R} \frac{\partial \zeta_{1}}{\partial \rho} .
$$

On the permeable membrane, use of (2.15) and (2.21) leads to

$$
\left.\begin{array}{l}
\Pi_{0}(1, \phi)=K_{0}, \\
\Pi_{1}(1, \phi)=K_{1}-\zeta_{0} \psi_{1}(1, \phi)+\frac{1}{R} \Re\left(\sum_{n=1}^{\infty} \frac{-i \xi_{n}^{\prime}(1) e^{i n \phi}}{n}\right)
\end{array}\right\}
$$

where $K_{0}$ and $K_{1}$ are arbitrary constants.

\section{The inner problem}

This involves the concentric region between the rotor and the permeable membrane. The length scale of the motion is typified by the annular gap $a-c$, which in general is small compared to $a$, while $\omega$ is $O(\Omega)$. In our viscometer, the ratio $(a-c) / a$ is a small parameter of $O\left(10^{-1}\right)$ say. Equation (2.6) is replaced by

$$
-\frac{1}{r} \frac{\partial(\Psi, \zeta)}{\partial(r, \theta)}=\frac{1}{R_{i}} \nabla^{2} \zeta, \quad \zeta=-\nabla^{2} \Psi
$$


where $R_{i}=(a-c) /(a)(c \omega) /(b \Omega) R$ is the effective Reynolds number and cylindrical polar coordinates are used. Since $c \ll b$, to a first approximation, the inner regime is described by the biharmonic equation

$$
\nabla^{4} \Psi=0
$$

We propose to use the same non-dimensionalization scheme as for the outer regime so that it is easier to match the boundary conditions on the permeable membrane. The biharmonic equation remains unchanged and holds in the region $c^{*} \leq r \leq 1$ where $c^{*}=c / a$. We will drop the asterisk on $c$ from now on.

If the membrane is impermeable there would be no interaction between the two regimes and the inner solution would be purely axi-symmetric. The permeability of the membrane will cause the solution to deviate from axi-symmetry, and we assume that this deviation is adequately modelled by considering the first order terms in $\gamma$ from the outer problem. Since the inner and outer solutions use two different coordinate systems, some care needs to be taken regarding the boundary conditions on the interface at $r=1=\rho$, where

$$
u_{\rho}(1, \phi)=\sqrt{J(1, \phi)} \frac{\partial \psi}{\partial \phi}(1, \phi)=u_{r}(1, \theta)=\frac{\partial \Psi}{\partial \theta}(1, \theta) .
$$

In the conversion of $\phi$ in terms of $\theta,(2.1)$ gives the following Fourier decomposition

$$
\begin{aligned}
e^{i n \phi}= & (-\gamma)^{n}+n(-\gamma)^{n-1}\left(1-\gamma^{2}\right) e^{i \theta} \\
& +\frac{n}{2}(-\gamma)^{n-2}\left(1-\gamma^{2}\right)\left[(n-1)-(n+1) \gamma^{2}\right] e^{i 2 \theta} \\
& +\frac{n}{6}(-\gamma)^{n-3}\left(1-\gamma^{2}\right)\left[(n-1)(n-2)-2\left(n^{2}-1\right) \gamma^{2}\right. \\
& \left.+(n+1)(n+2) \gamma^{4}\right] e^{i 3 \theta}+\cdots
\end{aligned}
$$

Applying (3.3) gives

$$
\psi_{1}(1, \phi)=\alpha_{0}+\alpha_{1} \cos \theta+\alpha_{2} \cos 2 \theta+\beta_{1} \sin \theta+\beta_{2} \sin 2 \theta+\cdots
$$

where

$$
\left.\begin{array}{l}
\alpha_{0}=b_{1} \gamma-b_{2} \gamma^{2} / 2+b_{3} \gamma^{3} / 3+\cdots \\
\alpha_{1}=\left(1-\gamma^{2}\right)\left(-b_{1}+\gamma b_{2}-\gamma^{2} b_{3}+\cdots\right) \\
\alpha_{2}=\left(1-\gamma^{2}\right)\left(-\gamma b_{1}-\left(1-3 \gamma^{2}\right) b_{2} / 2+\gamma\left(1-2 \gamma^{2}\right) b_{3}+\cdots\right) \\
\beta_{1}=\left(1-\gamma^{2}\right)\left(a_{1}-\gamma a_{2}+\gamma^{2} a_{3}+\cdots\right) \\
\beta_{2}=\left(1-\gamma^{2}\right)\left(\gamma a_{1}+\left(1-3 \gamma^{2}\right) a_{2} / 2-\gamma\left(1-2 \gamma^{2}\right) a_{3}+\cdots\right)
\end{array}\right\}
$$


with obvious extensions to higher harmonics. From (2.19), (2.24) and (2.32) we have

$$
\begin{aligned}
\psi_{1}(1, \phi) & =A \cos \phi+\Re\left\{\sum_{n=1}^{\infty} \chi_{n}(1) e^{i n \phi}\right\} \\
& =\mathfrak{N}\left\{\sum_{n=1}^{\infty} E_{n} e^{i n \phi}\right\} \\
& =\sum_{n=1}^{\infty}\left[a_{n} \sin (n \phi)-b_{n} \cos (n \phi)\right] / n .
\end{aligned}
$$

This is consistent with neglect of terms of $O\left(\gamma^{2}\right)$ in the outer problem. We now assume for the inner problem that the stream function on $r=1$ is completely given by $\Psi(1, \theta)=\psi_{0}(1)+\gamma \psi_{1}(1, \phi)$, hence

$$
\Psi(1, \theta)=-A / 2+K+\gamma \alpha_{0}+\gamma \sum_{n=1}^{\infty}\left[\alpha_{n} \cos n \theta+\beta_{n} \sin n \theta\right] .
$$

The other boundary conditions consist of a no-slip condition,

$$
\frac{\partial \Psi}{\partial r}(1, \theta)=0
$$

and on the rotor

$$
\frac{\partial \Psi}{\partial r}(c, \theta)=-\bar{U}, \quad \Psi(c, \theta)=0,
$$

where $\bar{U}=c \omega /(b \Omega)$. Equations (3.6) - (3.8) together with (3.1) completely specify the inner problem and $\gamma$ is now simply a predetermined constant. Since $r=c$ is a streamline, we have arbitrarily put $\Psi(c, \theta)=0$.

It is obvious, from the boundary conditions, that $\Psi$ may be decomposed into a component that is axi-symmetric and one that is non-symmetric. We, therefore, let

$$
\Psi(r, \theta)=\Psi_{0}(r)+\Psi_{1}(r, \theta)
$$

where $\Psi_{0}(r)$ satisfies

$$
\left.\begin{array}{rlrl}
\Psi_{0}(1) & =K_{2}, & \Psi_{0}(c) & =0, \\
\frac{\partial \Psi_{0}}{\partial r}(c) & =-\bar{U}, & \frac{\partial \Psi_{0}}{\partial r}(1) & =0,
\end{array}\right\}
$$

where $K_{2}=-A / 2+K+\gamma \alpha_{0}$, and the non-symmetric component satisfies

$$
\left.\begin{array}{l}
\Psi_{1}(1, \theta)=\gamma \sum_{n=1}^{\infty}\left(\alpha_{n} \cos n \theta+\beta_{n} \sin n \theta\right), \\
\Psi_{1}(c, \theta)=0=\frac{\partial \Psi_{1}}{\partial r}(1, \theta)=\frac{\partial \Psi_{1}}{\partial r}(c, \theta) .
\end{array}\right\}
$$


The axi-symmetric component is the solution for viscosity dominated Couette flow between rotating cylinders and has solution given by

$$
\Psi_{0}(r)=\frac{c \bar{U}}{1-c^{2}}\left(\frac{1}{2} r^{2}-\log (r / c)\right)-\frac{c^{3} \bar{U}}{2\left(1-c^{2}\right)},
$$

and

$$
K_{2}=\frac{1}{2} c \bar{U}+\frac{c \bar{U}}{1-c^{2}} \log c
$$

which determines $K$, also. Equation (3.11) suggests that $\Psi_{1}$ is, in general, part of a Fourier decomposition in $\theta$. Hence, it is sufficient to seek a solution of the form

$$
\Psi_{1}(r, \theta)=\Re\left(\sum_{n=1}^{\infty} \Upsilon_{n}(r) e^{i n \theta}\right)
$$

satisfying

$$
\begin{aligned}
& \Upsilon_{n}(1)=\gamma c_{n}, \quad c_{n}=\alpha_{n}-i \beta_{n}, \\
& \Upsilon_{n}(c)=0=\frac{\partial \Upsilon_{n}}{\partial r}(1)=\frac{\partial \Upsilon_{n}}{\partial r}(c) .
\end{aligned}
$$

$\Upsilon_{n}(r)$ satisfies

$$
r^{4} \Upsilon_{n}^{\prime \prime \prime \prime}+2 r^{3} \Upsilon_{n}^{\prime \prime \prime}-\left(2 n^{2}+1\right) r^{2} \Upsilon_{n}^{\prime \prime}+\left(2 n^{2}+1\right) r \Upsilon_{n}^{\prime}+n^{2}\left(n^{2}-4\right) \Upsilon_{n}=0
$$

which has solution sets $\left\{r, r \ln r, r^{-1}, r^{3}\right\}$ and $\left\{r^{n}, r^{-n}, r^{n+2}, r^{-n+2}\right\}$ when $n=1$ and $n \neq 1$, respectively. The solutions for the first two harmonics are

$$
\Upsilon_{1}(r)=\gamma c_{1}\left(\eta_{1} r+\eta_{2} r \ln r+\eta_{3} / r+\eta_{4} r^{3}\right)
$$

and

$$
\Upsilon_{2}(r)=\gamma c_{2}\left(\eta_{5} r^{2}+\eta_{6} / r^{2}+\eta_{7} r^{4}+\eta_{8}\right)
$$

where

$$
\begin{aligned}
\eta_{1} & =\frac{1}{4}\left[\left(3-c^{2}\right)\left(1-c^{2}\right)+2\left(3-c^{4}\right) \ln c\right] / d_{1}, & \eta_{2} & =-\frac{1}{2}\left(c^{2}+3\right)\left(1-c^{2}\right) / d_{1}, \\
\eta_{3} & =-\frac{1}{4} c^{2}\left[3\left(1-c^{2}\right)+2 c^{2} \ln c\right] / d_{1}, & \eta_{4} & =\frac{1}{4}\left(1-c^{2}-2 \ln c\right) / d_{1}, \\
\eta_{5} & =2\left(1+c^{2}+c^{4}\right) / d_{2}, & \eta_{6} & =2 c^{4} / d_{2}, \\
\eta_{7} & =-\left(1+c^{2}\right) / d_{2}, & \eta_{8} & =-c^{2}\left(4+c^{2}+c^{4}\right) / d_{2},
\end{aligned}
$$


and

$$
d_{1}=\left(1-c^{2}\right)\left[1-c^{2}+\left(1+c^{2}\right) \ln c\right], \quad d_{2}=\left(1-c^{2}\right)^{3} .
$$

Alternatively, we have

$$
\begin{aligned}
\Psi_{1}(r, \theta)= & \gamma\left(\eta_{1} r+\eta_{2} r \ln r+\eta_{3} / r+\eta_{4} r^{3}\right)\left(\alpha_{1} \cos \theta+\beta_{1} \sin \theta\right) \\
& +\gamma\left(\eta_{5} r^{2}+\eta_{6} / r^{2}+\eta_{7} r^{4}+\eta_{8}\right)\left(\alpha_{2} \cos 2 \theta+\beta_{2} \sin 2 \theta\right),
\end{aligned}
$$

for terms up to the second harmonic in $\theta$ only. As expected, $\Psi_{1}(r, \theta) \rightarrow 0$ if $\gamma$ is allowed to tend to zero.

As is well known for viscosity dominated flow, the pressure and vorticity are conjugate harmonic functions; thus, using

$$
\frac{\partial P}{\partial \theta}=\frac{r}{R} \frac{\partial \zeta}{\partial r}, \quad \zeta=-\nabla^{2} \Psi,
$$

the pressure distribution for terms up to the first and second harmonics is given by

$$
\begin{aligned}
P(r, \theta)= & \frac{\gamma}{R}\left[\left(\frac{2 \eta_{2}}{r}-8 r \eta_{4}\right)\left(\alpha_{1} \sin \theta-\beta_{1} \cos \theta\right)\right. \\
& \left.-\left(12 \eta_{7} r^{2}+\frac{4 \eta_{8}}{r^{2}}\right)\left(\alpha_{2} \sin 2 \theta-\beta_{2} \cos 2 \theta\right)\right]+K_{3},
\end{aligned}
$$

where $K_{3}$ is a constant. The torque (per unit length) exerted on the inner cylinder about its centre is given by $c^{2} \int_{0}^{2 \pi} \tau_{r \theta}(c, \theta) d \theta$ where $\tau_{r \theta}(c, \theta)$ is the non-dimensionalized shear stress on $r=c$. Since no contribution is made by $\Psi_{1}(r, \theta)$, the non-dimensional torque is given by

$$
T_{c}=-\frac{4 \pi}{1-c^{2}} .
$$

Setting $\delta=(1-c) / c$ as the clearance ratio, (3.16) yields

$$
T_{c}=-\frac{2 \pi}{\delta}\left[1+\frac{3 \delta}{2}+O\left(\delta^{2}\right)\right]
$$

in the limit as $\delta \rightarrow 0$, which agrees with [3] (equation (78)) for the concentric case.

\section{A solution for small $\lambda_{n}$}

We consider briefly some of the parameters of the problem. In practical terms, the annular gap between the rotor and the slotted sleeve is of the order of a centimetre. 
Typically, the sleeve may have an internal radius of 25 millimetres; this may be varied in order to see the effects on the flow through the sleeve. In MKS units we have based our analysis on a rotor of radius 0.019 metres, and 0.03 and 0.15 metres, respectively, for the sleeve and the rotating outer cylinder. For these values, $|\gamma|$ increases monotonically with the eccentricity, which is defined as $\bar{\epsilon}=a \epsilon /(b-a)$, but remains less than 0.15 for $0 \leq \bar{\epsilon} \leq 5 / 8$ so that the perturbation analysis may still be used when $\bar{\epsilon}$ is not much less than 1 (for example, in Figure 3 where $\bar{\epsilon}=1 / 2$, $|\gamma|=0.101)$. We note that the radius of the outer cylinder is chosen so that the formation of eddies is seen.

The closed form of the outer solution given by $\psi_{1}(\rho, \phi)$ does not convey much information about the flow pattern, but if $\sqrt{A R}$ is small, the Bessel functions may be approximated by their power series representation and in this limit we can get an idea of the flow field. Thus, we have that

$$
I_{ \pm \mu_{n}}\left(\lambda_{n} \rho\right) \sim \frac{\left(\lambda_{n} \rho / 2\right)^{ \pm \mu_{n}}}{\Gamma\left(1 \pm \mu_{n}\right)}+\frac{\left(\lambda_{n} \rho / 2\right)^{2 \pm \mu_{n}}}{\Gamma\left(2 \pm \mu_{n}\right)}+\cdots,
$$

or more precisely, for $n=1$ or 2 ,

$$
\begin{aligned}
I_{\mu_{1}}\left(\lambda_{1} \rho\right) & =\sigma_{11}(\rho) \delta+\sigma_{12}(\rho, \delta) \delta^{3}+O\left(\delta^{5}\right), \\
I_{-\mu_{1}}\left(\lambda_{1} \rho\right) & =\sigma_{13}(\rho) \delta+\sigma_{14}(\rho, \delta) \delta^{3}+O\left(\delta^{5}\right), \\
I_{\mu_{2}}\left(\lambda_{2} \rho\right) & =\sigma_{21}(\rho) \delta^{2}+\sigma_{22}(\rho, \delta) \delta^{4}+O\left(\delta^{6}\right), \\
I_{-\mu_{2}}\left(\lambda_{2} \rho\right) & =\sigma_{23}(\rho)+\sigma_{24}(\rho, \delta) \delta^{2}+O\left(\delta^{4}\right),
\end{aligned}
$$

where $\delta=\sqrt{i \overline{A R}}$ and

$$
\begin{aligned}
& \sigma_{11}(\rho)=\rho / 2, \\
& \sigma_{12}(\rho, \delta)=\frac{1}{4} \rho\left(1-\gamma^{\prime}+\rho^{2} / 4-\log (\rho \delta / 2)\right), \\
& \sigma_{13}(\rho)=1 / \rho+\rho / 2 \\
& \sigma_{14}(\rho, \delta)=\frac{1}{4} \rho^{-1}+\frac{1}{2}(1 / \rho+\rho / 2)\left[\gamma^{\prime}+\log (\rho \delta / 2)\right]+\rho^{3} / 16 \\
& \sigma_{21}(\rho)=\rho^{2} / 4, \\
& \sigma_{22}(\rho, \delta)=\frac{1}{4} \rho^{2}\left[3 / 4-\gamma^{\prime} / 2+\rho^{2} / 6-\frac{1}{2} \log (\rho \delta / \sqrt{2})\right], \\
& \sigma_{23}(\rho)=-1 / \rho^{2}, \\
& \sigma_{24}(\rho, \delta)=1 / 2+\frac{1}{2} \rho^{-2}\left[3 / 4-\gamma^{\prime}-\log (\rho \delta / \sqrt{2})\right]+\rho^{2} / 4 .
\end{aligned}
$$

In $\sigma_{12}, \sigma_{14}, \sigma_{22}$ and $\sigma_{24}$ the $\log \delta$ terms have not been separated out and $\gamma^{\prime}$ denotes Euler's constant. 
We are now in a position to obtain the constants $A_{n}, B_{n}, C_{n}$ and $D_{n}$ of (2.25) and (2.26) for $n=1$ or 2 in powers of $\delta$. The expansions are obtained using the symbolic algebraic package Maple (version V Release 2). For a description of the package see Char et al. [2]. Briefly, in the outer regime, we write Maple codes to obtain expansions for the Bessel functions and integrals contained in (2.25) and (2.26), as well as to store the expansions for the stream function and pressure. Using the codes as input files to Maple, the output files so obtained contain all the information required to write out the form of the remaining expressions in this section. Thus, the constant $A_{1}$, which is complex, is first expanded as $A_{11}+\delta^{2} A_{12}$, where $A_{12}$ contains $\log \delta$ terms as well. Denoting the real and imaginary parts by using a third suffix $r$ and $i$ respectively, and substituting for $\delta$ in terms of $A R$, we obtain $A_{1}=\left[A_{11 r}-A R A_{12 i}\right]+i\left[A_{11 i}+A R A_{12 r}\right]$ with neglect of terms of $O\left(R^{2} \log R, R^{2}\right)$, and $A_{12 r}$ and $A_{12 i}$ still contain $\log R$ terms. The other constants are decomposed in a similar manner. Next, the substitution of $\delta=\sqrt{i A R}$ into $\sigma_{12}, \sigma_{14}, \sigma_{22}$ and $\sigma_{24}$ leads to the complex quantities $\sigma_{12 r}+i \sigma_{12 i}$, $\sigma_{14 r}+i \sigma_{14 i}, \sigma_{22 r}+i \sigma_{22 i}$ and $\sigma_{24 r}+i \sigma_{24 i}$ respectively. Thus, the first two terms in the summation in (2.19) are given by

$$
\begin{aligned}
\chi_{n}(\rho) e^{i n \phi}=\cos n \phi & {\left[\rho^{n} A_{n 1 r}-A R \rho^{n} A_{n 2 i}+\rho^{-n} B_{n 1 r}-A R \rho^{-n} B_{n 2 i}-\omega_{n 1}(\rho) C_{n 1 r}\right.} \\
& -\omega_{n 3}(\rho) D_{n 1 r}+A R\left\{\omega_{n 1}(\rho) C_{n 2 i}+\omega_{n 2 i}(\rho) C_{n 1 r}+\omega_{n 2 r}(\rho) C_{n 1 i}\right. \\
& \left.\left.+\omega_{n 3}(\rho) D_{n 2 i}+\omega_{n 4 i}(\rho) D_{n 1 r}+\omega_{n 4 r}(\rho) D_{n 1 i}\right\}\right] \\
+\sin n & \phi\left[-\rho^{n} A_{n 1 i}-A R \rho^{n} A_{n 2 r}-\rho^{-n} B_{n 1 i}-A R \rho^{-n} B_{n 2 r}+\omega_{n 1}(\rho) C_{n 1 i}\right. \\
& +\omega_{n 3}(\rho) D_{n 1 i}+A R\left\{\omega_{n 1}(\rho) C_{n 2 r}+\omega_{n 2 r}(\rho) C_{n 1 r}-\omega_{n 2 i}(\rho) C_{n 1 i}\right. \\
& \left.\left.+\omega_{n 3}(\rho) D_{n 2 r}+\omega_{n 4 r}(\rho) D_{n 1 r}-\omega_{n 4 i}(\rho) D_{n 1 i}\right\}\right],
\end{aligned}
$$

for $n=1$ or 2 . In general,

$$
\omega_{n k .}=\frac{1}{2 n} \int_{1}^{\rho}\left(\frac{\rho^{n}}{s^{n-1}}-\frac{s^{n+1}}{\rho^{n}}\right) \sigma_{n k .}(s) d s, \quad n=1,2, k=1,2,
$$

and

$$
\omega_{n k .}=\frac{1}{2 n} \int_{\rho}^{\beta}\left(\frac{s^{n+1}}{\rho^{n}}-\frac{\rho^{n}}{s^{n-1}}\right) \sigma_{n k .}(s) d s, \quad n=1,2, k=3,4 .
$$

The last two equations hold with or without a third suffix for $\omega_{n k}$ and $\sigma_{n k}$. The inner regime is similarly solved using a set of Maple codes.

The flux condition on the membrane implies

$$
f(\phi ; \gamma)=-k[\Pi(1, \phi)-P(1, \theta)],
$$

where $k$ depends on the porosity of the membrane and is assumed known, and the pressure distributions are given by (2.36) and (3.15). If $f_{1}(\phi)$ is given by (2.18) up 
to the $N$ th harmonic in $\phi$, there are $2 N$ unknowns. Expressing $P(1, \theta)$ in terms of $\phi$ and collecting the coefficients of the first $N$ harmonics in (4.6) allows us to determine $a_{n}, b_{n}$ for $n=1, \ldots, N$. The actual solution for these constants, as well as the contour plots in the next section, are done using Maple routines. Ten significant digits are kept in all the numerical computations. The output expressions for the inner and outer stream functions satisfy all the boundary conditions imposed with a maximum error of $O\left(10^{-6}\right)$. For the case $\bar{\epsilon}=7 / 16, R=3.75, k=0.8$ and $\bar{U}=1.25$, we display the expressions for $\psi_{0}(\rho)+\gamma \psi_{1}(\rho, \phi)$ and $\Psi(r, \theta)$ below, but only four significant digits are retained here.

$$
\begin{aligned}
\psi(\rho, \phi) & =0.2422 \log \rho-0.1211 \rho^{2}-0.08735 \\
& +\gamma\left[\left(0.4016 \rho^{3}+2.625 \rho-3.027 / \rho-6.856 \rho \log \rho\right) \cos \phi\right. \\
& +\left(0.02544 \rho^{3}+\rho(0.9299-0.9615 \log \rho)+0.04470 / \rho\right)\left(a_{1} \sin \phi-b_{1} \cos \phi\right) \\
& +\left(0.001716 \rho^{4}-0.06536 \rho^{2}+0.6256-0.06193 / \rho^{2}\right)\left(a_{2} \sin 2 \phi-b_{2} \cos 2 \phi\right) \\
& +R\left\{\left(-0.001608 \rho^{5}+\rho^{3}(0.2269 \log \rho-0.4451)+\rho(0.2673+1.029 \log \rho\right.\right. \\
& \left.\left.+0.4152(\log \rho)^{2}\right)+0.1794 / \rho\right) \sin \phi \\
& +\left(0.0002567 \rho^{5}+\rho^{3}(0.06167-0.03219 \log \rho)-\rho(0.04181+0.1324 \log \rho\right. \\
& \left.\left.+0.05822(\log \rho)^{2}\right)-0.02011 / \rho\right)\left(b_{1} \sin \phi+a_{1} \cos \phi\right) \\
& +\left(0.00002598 \rho^{6}-\rho^{4}(0.0002078 \log \rho+0.002083)\right. \\
& \left.+\rho^{2}(0.07576 \log \rho-0.08683)+0.07576 \log \rho+0.1042-0.01527 / \rho^{2}\right) \\
& \left.\left.\times\left(b_{2} \sin 2 \phi+a_{2} \cos 2 \phi\right)\right\}\right], \quad 1 \leq \rho \leq 4.883
\end{aligned}
$$

and

$$
\begin{aligned}
\Psi(r, \theta)= & 0.6609 r^{2}-0.2651-1.322 \log (1.579 r) \\
& +\left(-15.37 r^{2}+10.54 r+41.40 r \log r+5.830 / r\right) \\
& \times(0.005925 \cos \theta-0.002475 \sin \theta) \\
& +\left(-6.523 r^{4}-8.519+14.54 r^{2}+1.498 / r^{2}\right) \\
& \times(-0.0008862 \cos 2 \theta+0.0003569 \sin 2 \theta), \quad 0.6333 \leq r \leq 1,
\end{aligned}
$$

where $a_{1}=0.02991, a_{2}=-0.003580, b_{1}=0.07168$, and $b_{2}=-0.009338$ and terms with numerical coefficients smaller than $10^{-8}$ have been neglected. To this approximation, terms of $O(R \log R)$ in $\psi_{1}(\rho, \phi)$ do not contribute to the outer solution.

\section{Results and discussion}

Since we have required $A R$ to be small, we are restricted to highly viscous fluids. Some common fluids permissible are $100 \%$ glycerol (by weight) and sucrose solutions 
at $20^{\circ} \mathrm{C}$, or glucose at $27^{\circ} \mathrm{C}$. For the contour plots given below, the contour interval is approximately 0.2 . The magnitudes in the outer regime being about one order of magnitude greater than those in the inner regime.

As a no-slip condition is imposed along the membrane, the flow through the membrane is given by the radial velocity alone; however, the profile plots obtained can be used to identify the salient features of the flow. Figure 2 represents the profile plots generated from the following sets of parameters:

1. $\bar{\epsilon}=3 / 16, R=3.75, k=0.3$;

2. $\bar{\epsilon}=3 / 16, R=3.75, k=0.8$;

3. $\bar{\epsilon}=7 / 16, R=1.00, k=0.8$; and

4. $\bar{\epsilon}=7 / 16, R=3.75, k=0.8$.

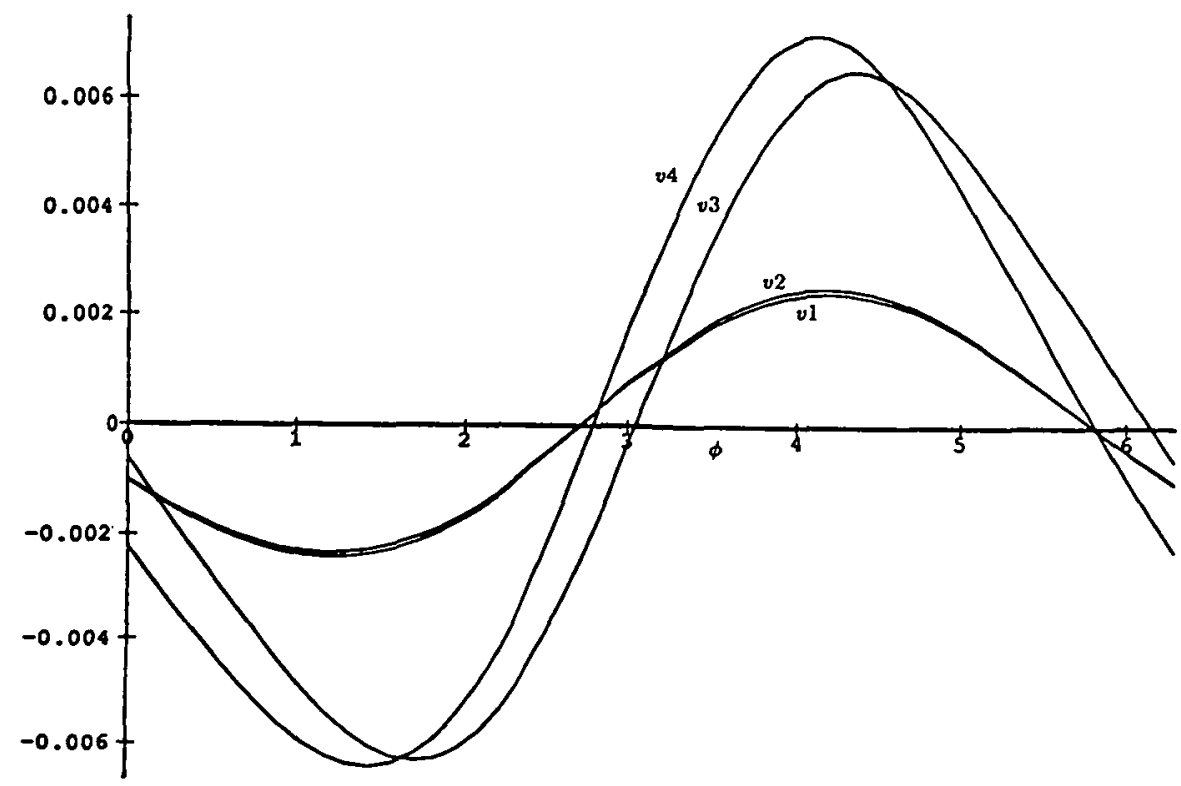

FIGURE 2. Profile plots for various radial velocities $(v 1, \bar{\epsilon}=3 / 16, R=3.75, k=0.3 ; v 2, \bar{\epsilon}=3 / 16$, $R=3.75, k=0.8 ; v 3, \bar{\epsilon}=7 / 16, R=1.00, k=0.8 ; v 4, \bar{\epsilon}=7 / 16, R=3.75, k=0.8$ ).

The profile plots clearly indicate that the regions of entry and exit are subdivided into two (unequal) regions along the membrane. While plots $v 1$ and $v 2$ show that varying the porosity of the membrane does not enhance greatly the flow through the membrane, $v 2$ and $v 4$ demonstrate that positioning the viscometer close to the outer bowl has the greatest influence on the through-flow, as one would expect. Furthermore, Figure 2 illustrates a phase shift in the velocity distribution as the Reynolds number is increased (cf. v3,v4). This gradual phase shift to the left, together with a slight increase in the speed of the through-flow, is found to be independent of eccentricity. 
Numerous trials have found that as $\bar{\epsilon}$ is increased the flow through the membrane is predominantly along the side closest to the outer bowl; however, Figure 3 illustrates that this dominance is retarded by increasing the speed of the inner rotor.
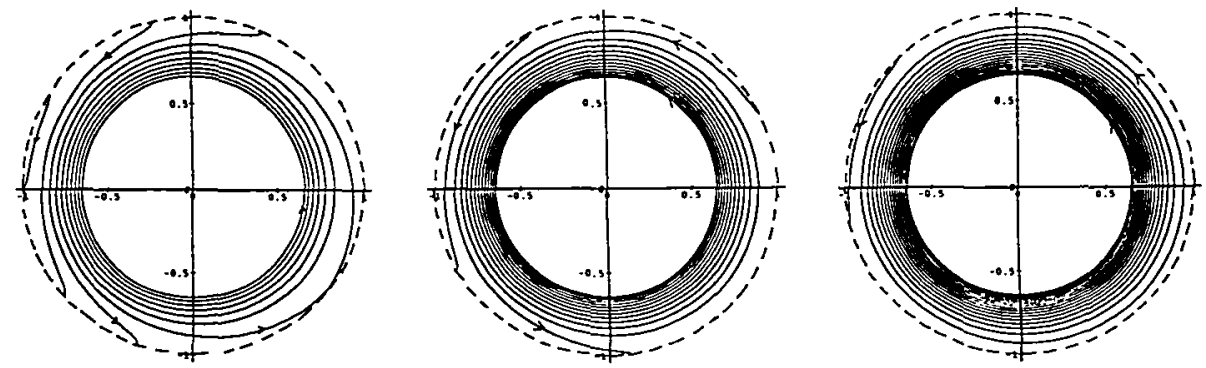

FIGURE 3. Streamlines through the annular region for (a) $\bar{U}=0.25$, (b) $\bar{U}=0.75$ (c) $\bar{U}=2.0$ with $\bar{\epsilon}=1 / 2, R=3.75, k=0.5$.

Figure 4 illustrates an eddy formation as $\bar{U}$ is increased when $\bar{\epsilon}=3 / 8$. While the presence of an eddy is found to be eccentricity dependent, the Reynolds number influences its location. This is exemplified in Figure 5 by noting that as the Reynolds number is increased, the eddies move in the direction opposite to that of the rotation of the outer bowl. Although no direct comparison can be made, we note that the displacement of the eddy centre with increasing Reynolds number has been observed in studies involving flows between eccentric cylinders (see [1,5]).
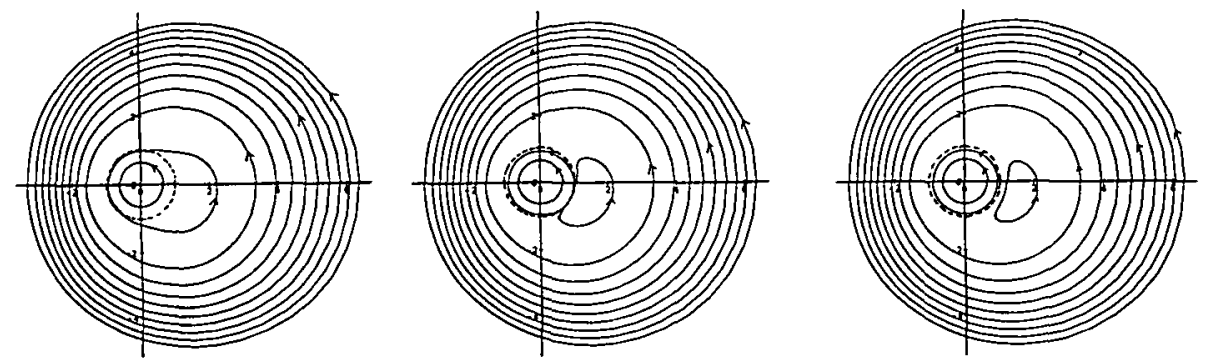

FigurE 4. An eddy formation with $\bar{\epsilon}=3 / 8, R=3.75, k=0.5$ for (a) $\bar{U}=1.20$ (b) $\bar{U}=1.23$ (c) $\bar{U}=1.25$.

\section{Conclusion}

For the geometrical restrictions placed on the system under study, we have shown that for highly viscous fluids the eccentricity has the greatest influence over both the radial velocity through the membrane and the presence of eddies, whose locations are 

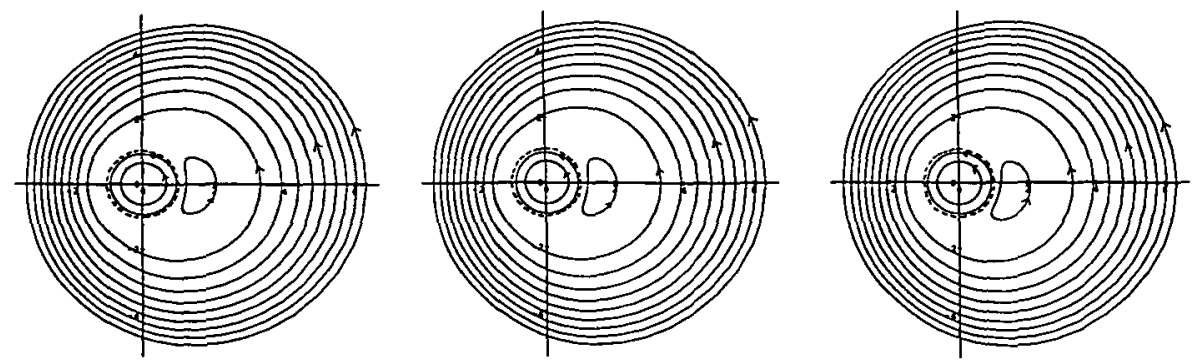

FIGURE 5. Streamlines in the entire flow regime for (a) $R=0.5$ (b) $R=1.5$ (c) $R=3.0$ with $\bar{\epsilon}=3 / 8, \bar{U}=1.25, k=0.5$.

influenced by the Reynolds number. Increasing the rotor speed appears to inhibit the entrainment of fluid through the membrane. This flow inhibition has an important consequence on the study of slurries. Assuming the flow pattern is relevant for slurries, experimental results will be less reliable if the inner rotor is maintained at a high rotation rate for prolonged periods, since the particle trapped inside the viscometer will tend to settle. Furthermore, we anticipate the presence of eddies close to the membrane will affect the entrainment process; however, for the present model, the radial velocity through the membrane is invariant to changes in the rotational speeds and, therefore, this assertion cannot be verified. The model clearly becomes invalid when a modified viscometer with large slots is considered. In this case it will be necessary to either relax the no-slip condition or use a numerical treatment, or both. We note that the computational complexity in this problem has been largely removed by use of an algebraic manipulative package.

\section{Acknowledgment}

We would like to thank the staff of the Curtin University Slurry Research Group for their assistance in providing the relevant facts about the viscometry apparatus considered in this paper.

\section{References}

[1] B. Y. Ballal and R. S. Rivlin, "Flow of a Newtonian fluid between eccentric rotating cylinders inertial effects", Arch. Rat. Mech. Anal. 62 (1977) 237-294.

[2] B. W. Char, K. O. Geddes, G. H. Gonnet, B. L. Leong, M. B. Monagan and S. M. Watt, First leaves: A tutorial introdution to Maple V (Springer, New York, 1992).

[3] R. C. DiPrima and J. T. Stuart, "Flow between eccentric rotating cylinders", J. Lubr. Tech., Trans. ASME. 94 (1972) 266-274. 
[4] I. J. Overend, R. R. Horsley, R. L. Jones and R. K. Vinycomb, “A new method for the measurement of rheological properties of settling slurries", Proc. IXth International Congress on Rheology, Mexico (1984) 583-590.

[5] A. San Andres and A. Z. Szeri, "Flow between eccentric rotating cylinders", J. App. Mech. 51 (1984) 869-878.

[6] G. N. Watson, A treatise on the theory of Bessel functions. 2nd ed. (Cambridge, London, 1944).

[7] W. W. Wood, "The asymptotic expansion at large Reynolds numbers for steady motion between non-coaxial rotating cylinders", J. Fluid. Mech. 3 (1957) 159-175.

[8] L. C. Woods, The theory of subsonic plane flow (Cambridge, London, 1961). 